\title{
Foreword by Guest Editor for the Special Issue on the 2010 ICUFN Conference
}

\author{
C. K. Toh
}

Published online: 26 February 2011

C) Springer Science+Business Media, LLC. 2011

Welcome to this special issue of Wireless Personal Communication Journal. We have selected a set of best papers from the 2010 ICUFN (International Conference on Ubiquitous Future Networks) Conference. All these papers have been extended and reviewed again by 3 independent reviewers. ICUFN is an annual international conference (www.icufn.org) technically co-sponsored by IEEE Communications Society and organized by the KICS (Korean Information \& Communications Society).

The first paper is entitled "Infoshare: Design and Implementation of Scalable Multimedia Signage Architecture for Wireless Ubiquitous Environment". The authors stated that communication disruptions are expected in a wireless environment and hence the there is a need for a generic architecture for a digital signage system using smart clients and XAML for expressing signage displays.

The second paper is entitled "Analytical Expression of Maximum Throughput for LongFrame Communications in One-way String Wireless Multihop Networks". The authors urged that there is currently no analytical expression for maximum throughput for long-frame communications in IEEE 802.11 Distributed Coordination (DCF) multihop networks. The authors validated their assumptions and analysis through further simulation results.

The partner selection problem for cooperative transmission is a recent topic of interest. The third paper- "Grouping Algorithm for Partner Selection in Cooperative Transmission" addresses this topic. The authors proposed a simple but optimal rate allocation algorithm for two cooperating nodes pairs and presented a grouping algorithm capable of reducing communication and computational overhead.

The fourth paper- "RAI: A High Throughput Routing Protocol for Multi-hop Multi-rate Ad Hoc Networks" addresses the problem of finding the appropriate rate and making the right routing decision to choose a high throughput route while avoiding potential link bottlenecks, thereby reducing packet drop rate. The authors showed through simulation results that their proposed Route Assessment Index (RAI) is effective and efficient.

C. K. Toh (ه)

ALICO Inc., Torrance, CA 90501, USA

e-mail: cktoh2003@yahoo.com 
Femto cells have recently been advocated for use in the subscriber's home environment. The authors stated that several sources of interferences will exist in a femtocellular network environment due to the density of femtocells and overlaid macrocells. The fifth paper- "Cost Effective Frequency Planning for Capacity Enhancement of Deployable Femtocellular Networks" addresses this issue and the authors proposed various frequency allocation schemes to mitigate interference as well as to increase the spectral efficiency.

The sixth paper on "Intersection-based Routing Protocol for VANETs" concerns vehicular ad hoc networks. The authors highlighted the challenges of routing in VANETs and proposed an intersection-based routing protocol that finds a minimum-delay path under different vehicular density conditions. The authors then compared their method with those of other solutions.

Finally, the last paper is entitled "Rate-control and Queueing of Backhaul Downstream Traffic for Mobile Wireless Systems". In the authors' view, existing legacy model considers only a queue at a base station (downstream traffic). Hence, they focused on queues at both base station and access routers and proposed a queue-aware rate control algorithm to avoid buffer overflow and underflow.

I would like to thank the Editor-in-chief (Professor Ramjee Prasad) and publishing assistant (Mrs. Cindy Zitter) for their support and help in realizing this special issue. Special thanks to all the authors for promptly revising their papers to the requirements of the reviewers. Thanks to the organizers of ICUFN conference, especially the Organizing Co-Chair (Professor Yeong Min Jang) and TPC Co-Chair (Professor Seung Hyong Rhee) for gathering quality papers for this special issue. I hope you will enjoy reading this special issue.

\section{Author Biography}

C. K. Toh received his Ph.D. in Computer Science from Cambridge University (1996) and earlier E.E. degrees from Manchester University (1991) and Singapore Polytechnic (1986). He was a visiting professor at YALE (2007), NCKU (Taiwan), Fudan (China), KTH (Sweden), KNU (Korea), and Osaka University (Japan). Since 2004, he has been an Honorary Full Professor at the University of Hong Kong. Previously, he was a tenured Chair Professor at the University of London and the Director of Research (Communication Systems) at TRW Tactical Systems Inc., USA. Earlier on, he led the DARPA Deployable Ad Hoc Networks program at Hughes Research. Dr. Toh had authored two books: (a) Ad Hoc Mobile Wireless Networks (Prentice Hall, ISBN 0130078174, 2001), and (b) Wireless ATM (Kluwer, ISBN 079239822X, 1997). He has consulted for various companies, including KDDI (Japan), Hughes (USA), and Mitsubishi (USA). Dr. Toh is a Fellow of the British Computer Society (BCS), New Zealand Computer Society (NZCS), Hong Kong Institution of Engineers (HKIE), and Institution of Electrical Engineers (IEE). He is a recipient of the 2005 IEEE Institution Kiyo Tomiyasu Technical Field Award (for pioneering contributions to communication protocols in ad hoc mobile networks) and 2009 IET Ambrose Fleming Medal. In 2009, he was named an IEEE Fellow and AAAS Fellow. 